\title{
Relation graphs of an association scheme based on attenuated spaces
}

\author{
Wen Liu • Kaishun Wang
}

Received: 25 October 2012 / Accepted: 18 February 2014 / Published online: 1 April 2014

(C) Springer Science+Business Media New York 2014

\begin{abstract}
The set of subspaces with a given dimension in an attenuated space has a structure of a symmetric association scheme, which is a generalization of both Grassmann schemes and bilinear forms schemes. In this paper, we focus on two families of relation graphs. Their full automorphism groups are completely determined. As a consequence, the classical results of the automorphism groups of Grassmann graphs and bilinear forms graphs are generalized.
\end{abstract}

Keywords Association scheme - Attenuated space - Relation graph · Full automorphism group

Mathematics Subject Classification (2010) 05E30

\section{Introduction}

Let $\mathbb{F}_{q}$ be a finite field with $q$ elements, where $q$ is a prime power. For two non-negative integers $n$ and $l$, suppose $\mathbb{F}_{q}^{(n+l)}$ denotes an $(n+l)$-dimensional row vector space over

W. Liu $\cdot$ K.Wang $(\varangle)$

School of Mathematical Sciences \& Lab. Math.Com. Sys., Beijing Normal University,

Beijing 100875, China

e-mail: wangks@bnu.edu.cn

W. Liu

Hebei Key Lab of Computational Mathematics \& Applications, and College of Math \& Info. Sciences, Hebei Normal University,

Shijiazhuang 050024, China 
$\mathbb{F}_{q}$. The set of all matrices over $\mathbb{F}_{q}$ of the form

$$
\left(\begin{array}{cc}
T_{11} & T_{12} \\
0 & T_{22}
\end{array}\right)
$$

where $T_{11}$ and $T_{22}$ are non-singular $n \times n$ and $l \times l$ matrices, respectively, forms a group under matrix multiplication, called the singular general linear group of degree $n+l$ over $\mathbb{F}_{q}$ and denoted by $G L_{n+l, n}\left(\mathbb{F}_{q}\right)$.

Let $P$ be an $m$-dimensional subspace of $\mathbb{F}_{q}^{(n+l)}$, denote also by $P$ an $m \times(n+l)$ matrix of rank $m$ whose rows span the subspace $P$ and call the matrix $P$ a matrix representation of the subspace $P$. The group $G L_{n+l, n}\left(\mathbb{F}_{q}\right)$ acts on $\mathbb{F}_{q}^{(n+l)}$ by the vector matrix multiplication. This action induces an action on the set of subspaces of $\mathbb{F}_{q}^{(n+l)}$, i.e., a subspace $P$ is carried by $T \in G L_{n+l, n}\left(\mathbb{F}_{q}\right)$ into the subspace $P T$. The vector space $\mathbb{F}_{q}^{(n+l)}$ together with this group action is called the $(n+l)$-dimensional singular linear space over $\mathbb{F}_{q}$. This concept was introduced in $[12,13]$.

For $1 \leq i \leq n+l$, let $e_{i}$ be the vector in $\mathbb{F}_{q}^{(n+l)}$ whose $i$ th coordinate is 1 and all other coordinates are 0 . Denote by $E$ the $l$-dimensional subspace of $\mathbb{F}_{q}^{(n+l)}$ generated by $e_{n+1}, e_{n+2}, \ldots, e_{n+l}$. An $m$-dimensional subspace $P$ of $\mathbb{F}_{q}^{(n+l)}$ is called a subspace of type $(m, k)$ if $\operatorname{dim}(P \cap E)=k$. The collection of all the subspaces of type $(m, 0)$ in $\mathbb{F}_{q}^{(n+l)}$ is the attenuated space, denoted by $A_{q}(n, l)$.

Denote by $X_{m}$ the set of all subspaces of type $(m, 0)$ in $\mathbb{F}_{q}^{(n+l)}$. For $0 \leq i \leq$ $\min \{m, n-m\}$ and $0 \leq j-i \leq \min \{m-i, j, l\}$, let

$$
R_{i, j-i}=\left\{(P, Q) \in X_{m} \times X_{m} \mid \operatorname{dim}\left(P^{\prime} \cap Q^{\prime}\right)=m-i, \operatorname{dim}(P \cap Q)=m-j\right\},
$$

where

$$
P=\left(\begin{array}{cc}
n & l \\
P^{\prime} & P^{\prime \prime}
\end{array}\right), \quad Q=\left(\begin{array}{cc}
n & l \\
Q^{\prime} & Q^{\prime \prime}
\end{array}\right)
$$

Then the configuration

$$
\mathfrak{X}_{m}=\left(X_{m},\left\{R_{i, j-i}\right\}_{0 \leq i \leq \min \{m, n-m\}, \quad 0 \leq j-i \leq \min \{m-i, j, l\}}\right)
$$

is a symmetric association scheme, and its intersection numbers are computed in [12]. Recently, Kurihara [8] computed its character table. This scheme is a common generalization of Grassmann schemes and bilinear forms schemes. We refer readers to $[1,3]$ for the general theory of association schemes.

If $l=0$, the relation graph $\left(X_{m}, R_{1,0}\right)$ of $\mathfrak{X}_{m}$ is the Grassmann graph $J_{q}(n, m)$. In 1949, Chow determined its full automorphism group.

Theorem 1.1 ([4]) Let $1<m<n-1$. Then

$$
\operatorname{Aut}\left(J_{q}(n, m)\right)= \begin{cases}P \Gamma L\left(n, \mathbb{F}_{q}\right), & \text { if } n \neq 2 m, \\ P \Gamma L\left(n, \mathbb{F}_{q}\right) .2, & \text { if } n=2 m .\end{cases}
$$


In [6], Fujisaki et al. determined the full automorphism group of the twisted Grassmann graph $\tilde{J}_{q}(2 e+1, e)$. This graph has the same spectrum as the Grassmann graph $J_{q}(2 e+1, e)$; see [9].

The relation graph $\left(X_{m}, R_{0,1}\right)$ is $\left[\begin{array}{l}n \\ m\end{array}\right]$ copies of the bilinear forms graph $H_{q}(m, l)$. In particular, if $m=n$, the relation graph $\left(X_{m}, R_{0,1}\right)$ is $H_{q}(m, l)$. In 1951 , Hua determined its full automorphism group.

Theorem 1.2 ([7]) Let $n$ and $l$ be two integers not less than 2. Then

$$
\operatorname{Aut}\left(H_{q}(n, l)\right)= \begin{cases}P \Gamma L\left(n+l, \mathbb{F}_{q}\right)_{E}, & \text { if } n \neq l, \\ P \Gamma L\left(n+l, \mathbb{F}_{q}\right)_{E} \cdot 2, & \text { if } n=l .\end{cases}
$$

In this paper, we focus on the two graphs $\left(X_{m}, R_{1,0}\right)$ and $\left(X_{m}, R_{1,0} \cup R_{0,1}\right)$, and determine their full automorphism groups.

\section{The graph $\left(X_{m}, R_{1,0}\right)$}

Since $\left(X_{1}, R_{1,0}\right)$ is the complete multipartite graph $K_{\left[\begin{array}{l}n \\ 1\end{array}\right]}\left(q^{l}\right)$, its full automorphism group is the wreath product $S_{q^{l}}<S_{\left[\begin{array}{l}n \\ 1\end{array}\right]}$.

In this section we always assume that $1<m \leq n-1$ and $l>0$. For each integer $k$ with $2 \leq k \leq m$, let $\Gamma^{(k)}$ denote the relation graph $\left(X_{k}, R_{1,0}\right)$ of $\mathfrak{X}_{k}$, where

$$
R_{1,0}=\left\{(P, Q) \in X_{k} \times X_{k} \mid \operatorname{dim}\left(P^{\prime} \cap Q^{\prime}\right)=\operatorname{dim}(P \cap Q)=k-1\right\} .
$$

By the proof of Lemma 3.1 in [12], $\left(X_{k}, R_{1,0}\right)$ is an arc-transitive graph.

Note that an $m$-dimensional subspace $P$ of form $(1)$ in $\mathbb{F}_{q}^{(n+l)}$ is a vertex of $\Gamma^{(m)}$ if and only if $\operatorname{rank}\left(P^{\prime}\right)=m$. Therefore, two vertices $P, Q$ of $\Gamma^{(m)}$ are adjacent if and only if their sum $P+Q$ is a subspace of type $(m+1,0)$.

Lemma 2.1 Let $P$ and $Q$ be two distinct vertices as in (1) of $\Gamma^{(m)}$. If $\operatorname{dim}\left(P^{\prime} \cap Q^{\prime}\right)=$ $m-i$ and $\operatorname{dim}(P \cap Q)=m-j$, then the distance of $P$ and $Q$ is

$$
\partial(P, Q)= \begin{cases}j & \text { if } i>0 \\ j+1 & \text { if } i=0\end{cases}
$$

In particular, the diameter of $\Gamma^{(m)}$ is $m+1$ if $l \geq m$, or $\min \{m, n-m+l\}$ if $l<m$.

Proof In the Grassmann graph $J_{q}(n+l, m)$, two vertices $x$ and $y$ are at distance $j$ if and only if $\operatorname{dim}(x \cap y)=m-j$. Since $\Gamma^{(m)}$ is a subgraph of $J_{q}(n+l, m)$, by $\operatorname{dim}(P \cap Q)=m-j$ we have $\partial(P, Q) \geq j$. Write $P \cap Q=W=\left(\begin{array}{cc}n & l \\ W^{\prime} & W^{\prime \prime}\end{array}\right)$ and 


$$
P=\left(\begin{array}{ll}
W^{\prime} & W^{\prime \prime} \\
\alpha_{1}^{\prime} & \alpha_{1}^{\prime \prime} \\
\vdots & \vdots \\
\alpha_{j-i}^{\prime} & \alpha_{j-i}^{\prime \prime} \\
\delta_{1}^{\prime} & \delta_{1}^{\prime \prime} \\
\vdots & \vdots \\
\delta_{i}^{\prime} & \delta_{i}^{\prime \prime}
\end{array}\right), \quad Q=\left(\begin{array}{ll}
W^{\prime} & W^{\prime \prime} \\
\alpha_{1}^{\prime} & \beta_{1}^{\prime \prime} \\
\vdots & \vdots \\
\alpha_{j-i}^{\prime} & \beta_{j-i}^{\prime \prime} \\
\gamma_{1}^{\prime} & \gamma_{1}^{\prime \prime} \\
\vdots & \vdots \\
\gamma_{i}^{\prime} & \gamma_{i}^{\prime \prime}
\end{array}\right)
$$

Case $1 i>0$.

If $j=i$, write

$$
P_{1}=\left(\begin{array}{ll}
W^{\prime} & W^{\prime \prime} \\
\gamma_{1}^{\prime} & \gamma_{1}^{\prime \prime} \\
\delta_{2}^{\prime} & \delta_{2}^{\prime \prime} \\
\delta_{3}^{\prime} & \delta_{3}^{\prime \prime} \\
\vdots & \vdots \\
\delta_{i}^{\prime} & \delta_{i}^{\prime \prime}
\end{array}\right), P_{2}=\left(\begin{array}{ll}
W^{\prime} & W^{\prime \prime} \\
\gamma_{1}^{\prime} & \gamma_{1}^{\prime \prime} \\
\gamma_{2}^{\prime} & \gamma_{2}^{\prime \prime} \\
\delta_{3}^{\prime} & \delta_{3}^{\prime \prime} \\
\vdots & \vdots \\
\delta_{i}^{\prime} & \delta_{i}^{\prime \prime}
\end{array}\right), \ldots, \quad P_{j-1}=\left(\begin{array}{ll}
W^{\prime} & W^{\prime \prime} \\
\gamma_{1}^{\prime} & \gamma_{1}^{\prime \prime} \\
\gamma_{2}^{\prime} & \gamma_{2}^{\prime \prime} \\
\vdots & \vdots \\
\gamma_{i-1}^{\prime} & \gamma_{i-1}^{\prime \prime} \\
\delta_{i}^{\prime} & \delta_{i}^{\prime \prime}
\end{array}\right),
$$

then $\left(P, P_{1}, \ldots, P_{j-1}, Q\right)$ is a path of length $j$ from $P$ to $Q$. Therefore $\partial(P, Q)=j$. If $j>i$, write

$$
\begin{aligned}
& P_{1}=\left(\begin{array}{ll}
W^{\prime} & W^{\prime \prime} \\
\alpha_{1}^{\prime} & \alpha_{1}^{\prime \prime} \\
\vdots & \vdots \\
\alpha_{j-i-2}^{\prime} & \alpha_{j-i-2}^{\prime \prime} \\
\alpha_{j-i-1}^{\prime} & \alpha_{j-i-1}^{\prime \prime} \\
\gamma_{1}^{\prime} & \gamma_{1}^{\prime \prime} \\
\delta_{1}^{\prime} & \delta_{1}^{\prime \prime} \\
\vdots & \vdots \\
\delta_{i}^{\prime} & \delta_{i}^{\prime \prime}
\end{array}\right), \quad\left(\begin{array}{ll}
W^{\prime} & W^{\prime \prime} \\
\alpha_{1}^{\prime} & \alpha_{1}^{\prime \prime} \\
\vdots & \vdots \\
\alpha_{j-i-2}^{\prime} & \alpha_{j-i-2}^{\prime \prime} \\
\alpha_{j-i}^{\prime} & \beta_{j-i}^{\prime \prime} \\
\gamma_{1}^{\prime} & \gamma_{1}^{\prime \prime} \\
\delta_{1}^{\prime} & \delta_{1}^{\prime \prime} \\
\vdots & \vdots \\
\delta_{i}^{\prime} & \delta_{i}^{\prime \prime}
\end{array}\right), \ldots, \quad P_{j-i}=\left(\begin{array}{ll}
W^{\prime} & W^{\prime \prime} \\
\alpha_{2}^{\prime} & \beta_{2}^{\prime \prime} \\
\vdots & \vdots \\
\alpha_{j-i-1}^{\prime} & \beta_{j-i-1}^{\prime \prime} \\
\alpha_{j-i}^{\prime} & \beta_{j-i}^{\prime \prime} \\
\gamma_{1}^{\prime} & \gamma_{1}^{\prime \prime} \\
\delta_{1}^{\prime} & \delta_{1}^{\prime \prime} \\
\vdots & \vdots \\
\delta_{i}^{\prime} & \delta_{i}^{\prime \prime}
\end{array}\right),
\end{aligned}
$$

then $\left(P, P_{1}, \ldots, P_{j-i}\right)$ is a path of length $j-i$. Since $\operatorname{dim}\left(P_{j-i}^{\prime} \cap Q^{\prime}\right)=\operatorname{dim}\left(P_{j-i} \cap\right.$ $Q)=m-i$, we have $\partial\left(P_{j-i}, Q\right)=i$. It follows that $\partial(P, Q)=j$. 
Case $2 i=0$.

The neighborhood of $P$ consists of vertices

$$
R=\left(\begin{array}{ll}
\bar{P}^{\prime} & \bar{P}^{\prime \prime} \\
\xi^{\prime} & \xi^{\prime \prime}
\end{array}\right)
$$

where $\bar{P}=\left(\begin{array}{ll}\bar{P}^{\prime} & \bar{P}^{\prime \prime}\end{array}\right)$ is an $(m-1)$-dimensional subspace of $P$ and $\xi^{\prime} \in \mathbb{F}_{q}^{(n)} \backslash P^{\prime}$.

If $W \subseteq \bar{P}$, then $\operatorname{dim}(R \cap Q)=m-j$ and $\operatorname{dim}\left(\left(\begin{array}{c}\bar{P}^{\prime} \\ \xi^{\prime}\end{array}\right) \cap Q^{\prime}\right)=m-1$. By Case 1, one obtains $\partial(R, Q)=j$. If $W \nsubseteq \bar{P}$, similarly we have $\partial(R, Q)=j+1$. Hence, $\partial(P, Q)=j+1$.

Write

$$
M=\left(\begin{array}{cccc}
m & n-m & l \\
I & 0 & 0
\end{array}\right)=\left(\begin{array}{cc}
M^{\prime} & M^{\prime \prime}
\end{array}\right) .
$$

Then $M$ is a vertex of $\Gamma^{(m)}$. Next we shall study the first and second subconstituents of $\Gamma^{(m)}$ with respect to $M$, denoted by $\Gamma_{1}(M)$ and $\Gamma_{2}(M)$, respectively.

Let $\Gamma_{i, j-i}(M)$ be the set of vertices $P$ of $\Gamma^{(m)}$ satisfying $(M, P) \in R_{i, j-i}$. By Lemma 3.1 the second subconstituent $\Gamma_{2}(M)$ has a partition

$$
\Gamma_{0,1}(M) \dot{\cup} \Gamma_{1,1}(M) \dot{\cup} \Gamma_{2,0}(M) .
$$

For simplicity, write

$$
\begin{aligned}
& P(U ; \alpha, \beta, \gamma)=\left(\begin{array}{ccc}
m & n-m & l \\
\alpha & 0 & 0 \\
\alpha & \beta & \gamma
\end{array}\right){ }_{1}^{t}, \\
& m n-m \quad l \\
& P\left(U ; \alpha_{1}, \beta_{1}, \gamma_{1} ; \alpha_{2}, \beta_{2}, \gamma_{2}\right)=\left(\begin{array}{ccc}
U & 0 & 0 \\
\alpha_{1} & \beta_{1} & \gamma_{1} \\
\alpha_{2} & \beta_{2} & \gamma_{2}
\end{array}\right)_{1}^{t},
\end{aligned}
$$

where $\operatorname{rank}(U)=t, \operatorname{rank}\left(\begin{array}{ll}\beta & \gamma\end{array}\right)=1$ and $\operatorname{rank}\left(\begin{array}{ll}\beta_{1} & \gamma_{1} \\ \beta_{2} & \gamma_{2}\end{array}\right)=2$. Then

$\Gamma_{1}(M)=\{P(W ; \alpha, \beta, \gamma) \mid \operatorname{rank}(W)=m-1, \beta \neq 0\}$,

$\Gamma_{0,1}(M)=\{P(W ; \alpha, 0, \gamma) \mid \operatorname{rank}(W)=m-1, \alpha \notin W, \gamma \neq 0\}$,

$\Gamma_{1,1}(M)=\left\{P\left(U ; \alpha_{1}, \beta_{1}, \gamma_{1} ; \alpha_{2}, 0, \gamma_{2}\right) \mid \operatorname{rank}(U)=m-2, \alpha_{2} \notin U, \beta_{1} \neq 0, \gamma_{2} \neq 0\right\}$,

$\Gamma_{2,0}(M)=\left\{P\left(U ; \alpha_{1}, \beta_{1}, \gamma_{1} ; \alpha_{2}, \beta_{2}, \gamma_{2}\right) \mid \operatorname{rank}(U)=m-2, \operatorname{rank}\left(\begin{array}{l}\beta_{1} \\ \beta_{2}\end{array}\right)=2\right\}$. 
Lemma 2.2 ([11, Corollary 1.9]) Let $0 \leq k \leq m \leq n$. Then the number of $m$ dimensional subspaces containing a given $k$-dimensional subspace in $\mathbb{F}_{q}^{(n)}$ is equal to $\left[\begin{array}{l}n-k \\ m-k\end{array}\right]$.

Lemma 2.3 (i) Let $P(W ; \alpha, 0, \gamma) \in \Gamma_{0,1}(M)$ and $P\left(W^{\prime} ; \alpha^{\prime}, \beta^{\prime}, \gamma^{\prime}\right) \in \Gamma_{1}(M)$. Then the two vertices are adjacent in $\Gamma^{(m)}$ if and only if $W=W^{\prime}$. In particular, each vertex in $\Gamma_{0,1}(M)$ has $q^{l+1}\left[\begin{array}{c}n-m \\ 1\end{array}\right]$ neighbors in $\Gamma_{1}(M)$;

(ii) Each vertex in $\Gamma_{1,1}(M)$ has $q^{2}$ neighbors in $\Gamma_{1}(M)$. Moreover, the induced subgraph on these $q^{2}$ vertices is the Cartesian product $K_{q} \square K_{q}$;

(iii) If $\Gamma_{2,0}(M) \neq \emptyset$, then each vertex in $\Gamma_{2,0}(M)$ has $(q+1)^{2}$ neighbors in $\Gamma_{1}(M)$.

Proof (i) Suppose $P(W ; \alpha, 0, \gamma)$ is adjacent to $P\left(W^{\prime} ; \alpha^{\prime}, \beta^{\prime}, \gamma^{\prime}\right)$. If $W \neq W^{\prime}$, by $\beta^{\prime} \neq 0$ and $\gamma \neq 0$ the dimension of $P(W ; \alpha, 0, \gamma)+P\left(W^{\prime} ; \alpha^{\prime}, \beta^{\prime}, \gamma^{\prime}\right)$ is $m+2$, a contradiction. The converse is immediate from $\alpha \notin W$ and $\beta^{\prime} \neq 0$. Therefore, the first statement is valid. Since $P\left(W ; \alpha^{\prime}, \beta^{\prime}, \gamma^{\prime}\right)$ is of type $(m, 0)$, the rank of $\left(\begin{array}{cc}W & 0 \\ \alpha^{\prime} & \beta^{\prime}\end{array}\right)$ is $m$. By Lemma 2.2 the subspace $\left(\begin{array}{cc}W & 0 \\ \alpha^{\prime} & \beta^{\prime}\end{array}\right)$ with $\beta^{\prime} \neq 0$ has $q\left[\begin{array}{c}n-m \\ 1\end{array}\right]$ choices. For a given subspace $\left(\begin{array}{cc}W & 0 \\ \alpha^{\prime} & \beta^{\prime}\end{array}\right)$, there are $q^{l}$ choices for $\gamma^{\prime}$. Hence, (i) holds.

(ii) Given a vertex $P\left(U ; \alpha_{1}, \beta_{1}, \gamma_{1} ; \alpha_{2}, 0, \gamma_{2}\right) \in \Gamma_{1,1}(M)$. We claim that this vertex is adjacent to $P(W ; \alpha, \beta, \gamma) \in \Gamma_{1}(M)$ if and only if $U \subseteq W$ and $P\left(W ; \alpha_{1}, \beta_{1}, \gamma_{1} ; \alpha_{2}, 0, \gamma_{2}\right)$ is a subspace of type $(m+1,0)$ containing $P(W ; \alpha, \beta, \gamma)$. Suppose $P(W ; \alpha, \beta, \gamma)$ is adjacent to $P\left(U ; \alpha_{1}, \beta_{1}, \gamma_{1} ; \alpha_{2}, 0, \gamma_{2}\right)$. If $U \nsubseteq W$, by $\beta_{1} \neq 0$ and $\gamma_{2} \neq 0$, the dimension of $P(W ; \alpha, \beta, \gamma)+$ $P\left(U ; \alpha_{1}, \beta_{1}, \gamma_{1} ; \alpha_{2}, 0, \gamma_{2}\right)$ is at least $m+2$, a contradiction. It follows that $U \subseteq W$. Then

$$
\begin{aligned}
& P(W ; \alpha, \beta, \gamma)+P\left(U ; \alpha_{1}, \beta_{1}, \gamma_{1} ; \alpha_{2}, 0, \gamma_{2}\right) \\
& \quad=P(W ; \alpha, \beta, \gamma)+P\left(W ; \alpha_{1}, \beta_{1}, \gamma_{1} ; \alpha_{2}, 0, \gamma_{2}\right),
\end{aligned}
$$

and so $P\left(W ; \alpha_{1}, \beta_{1}, \gamma_{1} ; \alpha_{2}, 0, \gamma_{2}\right)$ is a subspace of type $(m+1,0)$ containing $P(W ; \alpha, \beta, \gamma)$. The converse is immediate. Hence, our claim is valid.

By Lemma 2.2 the number of $(m-1)$-subspaces $W$ in $\mathbb{F}_{q}^{(m)}$ containing $U$ is $q+1$. Observe that $P\left(W ; \alpha_{1}, \beta_{1}, \gamma_{1} ; \alpha_{2}, 0, \gamma_{2}\right)$ is of type $(m+1,0)$ if and only if $W \neq\left(\begin{array}{c}U \\ \alpha_{2}\end{array}\right)$. Then $W$ has $q$ choices. For a fixed $W$, by Lemma 2.2 again $P(W ; \alpha, \beta, \gamma)$ has $q$ choices, and each vertex is uniquely determined by the subspace $(\beta, \gamma)$. Moreover, the induced subgraph on these $q$ vertices is $K_{q}$. Pick two distinct vertices $P\left(W_{1} ; \alpha, \beta, \gamma\right)$ and $P\left(W_{2} ; \alpha^{\prime}, \beta^{\prime}, \gamma^{\prime}\right)$ in the common neighborhood of $M$ and $P\left(U ; \alpha_{1}, \beta_{1}, \gamma_{1} ; \alpha_{2}, 0, \gamma_{2}\right), W_{1} \neq W_{2}$. Since the two vertices are adjacent if and only if $(\beta, \gamma)$ and $\left(\beta^{\prime}, \gamma^{\prime}\right)$ are the same subspace. Therefore, (ii) holds.

The proof of (iii) is similar to that of (ii), and will be omitted. 
For a subspace $W$ of type $(m-1,0)$ in $\mathbb{F}_{q}^{(n+l)}$, let $C(W)$ be the set of all vertices of $\Gamma^{(m)}$ containing $W$. For convenience we denote by $\Delta$ the induced subgraph of $\Gamma^{(m)}$ on $C(W)$.

Lemma 2.4 Let $W$ be a subspace of type $(m-1,0)$ in $\mathbb{F}_{q}^{(n+l)}$. Then $\Delta$ is isomorphic to the complete multipartite graph $K_{\left[\begin{array}{c}n-m+1 \\ 1\end{array}\right]}\left(q^{l}\right)$.

Proof Let $W=\left(\begin{array}{cc}n & l \\ W^{\prime} & W^{\prime \prime}\end{array}\right)$. Then $C(W)$ consists of vertices

$$
\left(\begin{array}{cc}
W^{\prime} & W^{\prime \prime} \\
\alpha^{\prime} & \alpha^{\prime \prime}
\end{array}\right)
$$

where $\operatorname{rank}\left(\begin{array}{c}W^{\prime} \\ \alpha^{\prime}\end{array}\right)=m$. By Lemma 2.2 the number of $m$-dimensional subspaces $\left(\begin{array}{c}W^{\prime} \\ \alpha^{\prime}\end{array}\right)$ in $\mathbb{F}_{q}^{(n)}$ is $\left[\begin{array}{c}n-m+1 \\ 1\end{array}\right]$. Since $\alpha^{\prime \prime}$ has $q^{l}$ choices, the subgraph $\Delta$ has $q^{l}\left[\begin{array}{c}n-m+1 \\ 1\end{array}\right]$ vertices.

For a given $m$-dimensional subspace $\left(\begin{array}{c}W^{\prime} \\ \alpha^{\prime}\end{array}\right)$ in $\mathbb{F}_{q}^{(n)}$, the vertices of form (4) form an independent set with $q^{l}$ vertices. Note that all these independent sets form a partition of $C(W)$. Since each vertex in an independent set is adjacent to any vertex in the remaining independent sets, the desired result follows.

Lemma 2.5 Let $1<m \leq n-1$ and $l>0$ with $(m, l, q) \neq(n-1,1,2)$. If an induced subgraph $\Delta$ of $\Gamma^{(m)}$ is isomorphic to $K_{\left[\begin{array}{c}n-m+1 \\ 1\end{array}\right]}\left(q^{l}\right)$, then $\Delta$ is a subgraph $C(W)$, where $W$ is a subspace of type $(m-1,0)$ in $\mathbb{F}_{q}^{(n+l)}$.

Proof Since $\Gamma^{(m)}$ is arc-transitive, we may assume that $\Delta$ contains $M$. Pick $X \in$ $\Delta \cap \Gamma_{1}(M)$, and write $M \cap X=W$. Then

$$
W=\left(\begin{array}{ccc}
m & n-m & l \\
W^{\prime} & 0 & 0
\end{array}\right), \quad X=P\left(W^{\prime} ; \alpha^{\prime}, \beta^{\prime}, \gamma^{\prime}\right) .
$$

We claim that $\Delta \cap\left(\Gamma_{1,1}(M) \cup \Gamma_{2,0}(M)\right)=\emptyset$. Suppose for the contradiction that this set is not empty.

Case $1 . m \neq n-1$ or $l \neq 1$.

Pick a vertex $P \in \Delta \cap\left(\Gamma_{1,1}(M) \cup \Gamma_{2,0}(M)\right)$. Then $P$ and $M$ have $q^{l+1}\left[\begin{array}{c}n-m \\ 1\end{array}\right]$ common neighbors in $\Delta$, a contradiction to Lemma 2.3 (ii), (iii).

Case 2. $m=n-1$ and $l=1$.

Note that $\Gamma_{2,0}(M)=\emptyset$. Pick a vertex $P \in \Delta \cap \Gamma_{1,1}(M)$. Then the induced subgraph on the common neighbors of $M$ and $P$ is $K_{q}(q)$, contradicting to Lemma 2.3 (ii).

Hence, our claim is valid. So $\Delta \cap \Gamma_{0,1}(M) \neq \emptyset$. Pick a vertex $P\left(W^{\prime \prime} ; \alpha^{\prime \prime}, 0, \gamma^{\prime \prime}\right) \in$ $\Delta \cap \Gamma_{0,1}(M)$. Since $\Delta$ is a complete multipartite graph, the vertices $X$ and $P\left(W^{\prime \prime} ; \alpha^{\prime \prime}, 0, \gamma^{\prime \prime}\right)$ are adjacent. By Lemma 2.3 (i), one obtains $W^{\prime}=W^{\prime \prime}$. It follows that $\Delta \cap \Gamma_{0,1}(M) \subseteq C(W)$. Similarly, we have $\Delta \cap \Gamma_{1}(M) \subseteq C(W)$. Hence, 
$\Delta \subseteq C(W)$. By Lemma 2.4 the subgraphs $\Delta$ and $C(W)$ have the same number of vertices, so $\Delta=C(W)$.

If $(m, l, q)=(n-1,1,2)$, then $\Gamma_{1,1}(M) \neq \emptyset$. Pick a vertex $P$ from this set. Then the induced subgraph on the common neighbors of $P$ and $M$ together with $P$ and $M$ is not the subgraph $C(W)$. But this subgraph is $K_{3}(2)$, so Lemma 2.5 does not hold in this special case.

Theorem 2.6 Let $1<m \leq n-1$ and $l>0$. Then

$$
\operatorname{Aut}\left(\Gamma^{(m)}\right)= \begin{cases}\operatorname{Sym}\left(2^{n}\right), & \text { if }(m, l, q)=(n-1,1,2), \\ P \Gamma L\left(n+l, \mathbb{F}_{q}\right)_{E}, & \text { if }(m, l, q) \neq(n-1,1,2)\end{cases}
$$

Proof If $(m, l, q)=(n-1,1,2)$, by [8, Theorem 4.1] the graph $\Gamma^{(n-1)}$ is isomorphic to the graph whose vertices are the lines in the affine space $A G\left(n, \mathbb{F}_{2}\right)$ and two distinct vertices are adjacent if their intersection is not the empty set. Since each line in $A G\left(n, \mathbb{F}_{2}\right)$ consists of two distinct points, $\Gamma^{(n-1)}$ is isomorphic to the Johnson graph $J\left(2^{n}, 2\right)$. Hence, its full automorphism group is the symmetric group $\operatorname{Sym}\left(2^{n}\right)$.

Now suppose $(m, l, q) \neq(n-1,1,2)$. We first prove that the result holds for $m=2$. Pick any automorphism $\tau$ of $\Gamma^{(2)}$. Then $\tau$ is a permutation on the set of lines and permutes the points of the attenuated space $A_{q}(n, l)$. By Deng and Li's result in [5], the automorphism $\tau$ can be extended to a collineation fixing $E$ of the projective space $P G\left(n+l, \mathbb{F}_{q}\right)$. By the fundamental theorem of the projective geometry (cf. [2] and [10, Theorem 2.23]), we have $\tau \in P \Gamma L\left(n+l, \mathbb{F}_{q}\right)_{E}$. Thus, Aut $\left(\Gamma^{(2)}\right) \subseteq P \Gamma L\left(n+l, \mathbb{F}_{q}\right)_{E}$. On the other hand, $P \Gamma L\left(n+l, \mathbb{F}_{q}\right)_{E} \subseteq \operatorname{Aut}\left(\Gamma^{(2)}\right)$. Hence, $\operatorname{Aut}\left(\Gamma^{(2)}\right)=P \Gamma L(n+$ $\left.l, \mathbb{F}_{q}\right)_{E}$.

Now let $m \geq 3$ and $\sigma_{m}$ be an automorphism of the graph $\Gamma^{(m)}$. By Lemmas 2.4 and 2.5, the automorphism $\sigma_{m}$ induces a permutation on the set $\{C(W) \mid$ $\left.W \in X_{m-1}\right\}$, and further induces a permutation $\sigma_{m-1}$ on $X_{m-1}$. For any two vertices $W_{m-1}, W_{m-1}^{\prime}$ of $\Gamma^{(m-1)}$, we can check that $W_{m-1}$ and $W_{m-1}^{\prime}$ are adjacent if and only if $C\left(W_{m-1}\right) \cap C\left(W_{m-1}^{\prime}\right) \neq \emptyset$. If $W_{m-1}$ and $W_{m-1}^{\prime}$ are adjacent, then $\sigma_{m}\left(C\left(W_{m-1}\right) \cap C\left(W_{m-1}^{\prime}\right)\right)=C\left(\sigma_{m-1}\left(W_{m-1}\right)\right) \cap C\left(\sigma_{m-1}\left(W_{m-1}^{\prime}\right)\right) \neq \emptyset$, which implies that the two vertices $\sigma_{m-1}\left(W_{m-1}\right)$ and $\sigma_{m-1}\left(W_{m-1}^{\prime}\right)$ are adjacent in $\Gamma^{(m-1)}$. Hence, $\sigma_{m-1} \in \operatorname{Aut}\left(\Gamma^{(m-1)}\right)$.

By induction, for each $3 \leq k \leq m$, the map $f_{k}: \sigma_{k} \longmapsto \sigma_{k-1}$ is a homomorphism from $\operatorname{Aut}\left(\Gamma^{(k)}\right)$ to $\operatorname{Aut}\left(\Gamma^{(k-1)}\right)$. We claim that $f_{k}$ is injective. Suppose $\sigma_{k-1}=\iota$, the identity permutation on $X_{k-1}$. For each vertex $W_{k}$ of $\Gamma^{(k)}$, there exist two vertices $W_{k-1}$ and $W_{k-1}^{\prime}$ of $\Gamma^{(k-1)}$ such that $W_{k}=W_{k-1}+W_{k-1}^{\prime}$. Since $\left\{W_{k}\right\}=C\left(W_{k-1}\right) \cap$ $C\left(W_{k-1}^{\prime}\right)$, we have $\left\{\sigma_{k}\left(W_{k}\right)\right\}=C\left(\sigma_{k-1}\left(W_{k-1}\right)\right) \cap C\left(\sigma_{k-1}\left(W_{k-1}^{\prime}\right)\right)=\left\{W_{k}\right\}$. Hence our claim is valid. It follows that $\left|\operatorname{Aut}\left(\Gamma^{(m)}\right)\right| \leq\left|\operatorname{Aut}\left(\Gamma^{(2)}\right)\right|$. Since $P \Gamma L\left(n+l, \mathbb{F}_{q}\right)_{E}$ is a subgroup of $\operatorname{Aut}\left(\Gamma^{(m)}\right)$, the desired result follows.

\section{The graph $\left(X_{m}, R_{1,0} \cup R_{0,1}\right)$}

Let $\Gamma_{w}^{(m)}$ denote the graph $\left(X_{m}, R_{0,1} \cup R_{1,0}\right)$ of the scheme $\mathfrak{X}_{m}$. Then $\Gamma_{w}^{(m)}$ is a subgraph of the Grassmann graph $J_{q}(n+l, m)$. If $l=0, \Gamma_{w}^{(m)}$ is the Grassmann graph $J_{q}(n, m)$; 
If $m=n$ and $l>0, \Gamma_{w}^{(m)}$ is the bilinear forms graph $H_{q}(m, l)$. Note that $\Gamma_{w}^{(m)}$ and $\Gamma^{(m)}$ have the same vertex set.

Lemma 3.1 Let $P$ and $Q$ be two vertices of $\Gamma_{w}^{(m)}$. Then $\partial(P, Q)=m-\operatorname{dim}(P \cap Q)$. In particular, the diameter of $\Gamma_{w}^{(m)}$ is $\min \{m, n-m+l\}$.

Proof Suppose $(P, Q) \in R_{i, j-i}$. Then $\operatorname{dim}(P \cap Q)=m-j$. Write $P \cap Q=$ $\left(\begin{array}{ll}W^{\prime} & W^{\prime \prime}\end{array}\right)$. Then $P$ and $Q$ are of form (3). Write

$$
P_{1}=\left(\begin{array}{cc}
W^{\prime} & W^{\prime \prime} \\
\alpha_{1}^{\prime} & \alpha_{1}^{\prime \prime} \\
\vdots & \vdots \\
\alpha_{j-i}^{\prime} & \alpha_{j-i}^{\prime \prime} \\
\gamma_{1}^{\prime} & \gamma_{1}^{\prime \prime} \\
\delta_{2}^{\prime} & \delta_{2}^{\prime \prime} \\
\delta_{3}^{\prime} & \delta_{3}^{\prime \prime} \\
\vdots & \vdots \\
\delta_{i}^{\prime} & \delta_{i}^{\prime \prime}
\end{array}\right), P_{2}=\left(\begin{array}{cc}
W^{\prime} & W^{\prime \prime} \\
\alpha_{1}^{\prime} & \alpha_{1}^{\prime \prime} \\
\vdots & \vdots \\
\alpha_{j-i}^{\prime} & \alpha_{j-i}^{\prime \prime} \\
\gamma_{1}^{\prime} & \gamma_{1}^{\prime \prime} \\
\gamma_{2}^{\prime} & \gamma_{2}^{\prime \prime} \\
\delta_{3}^{\prime} & \delta_{3}^{\prime \prime} \\
\vdots & \vdots \\
\delta_{i}^{\prime} & \delta_{i}^{\prime \prime}
\end{array}\right), \ldots, P_{i}=\left(\begin{array}{cc}
W^{\prime} & W^{\prime \prime} \\
\alpha_{1}^{\prime} & \alpha_{1}^{\prime \prime} \\
\vdots & \vdots \\
\alpha_{j-i}^{\prime} & \alpha_{j-i}^{\prime \prime} \\
\gamma_{1}^{\prime} & \gamma_{1}^{\prime \prime} \\
\gamma_{2}^{\prime} & \gamma_{2}^{\prime \prime} \\
\vdots & \vdots \\
\gamma_{i-1}^{\prime} & \gamma_{i-1}^{\prime \prime} \\
\gamma_{i}^{\prime} & \gamma_{i}^{\prime \prime}
\end{array}\right),
$$

$$
P_{i+1}=\left(\begin{array}{cc}
W^{\prime} & W^{\prime \prime} \\
\alpha_{1}^{\prime} & \beta_{1}^{\prime \prime} \\
\alpha_{2}^{\prime} & \alpha_{2}^{\prime \prime} \\
\alpha_{3}^{\prime} & \alpha_{3}^{\prime \prime} \\
\vdots & \vdots \\
\alpha_{j-i-1}^{\prime} & \alpha_{j-i-1}^{\prime \prime} \\
\alpha_{j-i}^{\prime} & \alpha_{j-i}^{\prime \prime} \\
\gamma_{1}^{\prime} & \gamma_{1}^{\prime \prime} \\
\vdots & \vdots \\
\gamma_{i}^{\prime} & \gamma_{i}^{\prime \prime}
\end{array}\right), \quad P_{i+2}=\left(\begin{array}{cc}
W^{\prime} & W^{\prime \prime} \\
\alpha_{1}^{\prime} & \beta_{1}^{\prime \prime} \\
\alpha_{2}^{\prime} & \beta_{2}^{\prime \prime} \\
\alpha_{3}^{\prime} & \alpha_{3}^{\prime \prime} \\
\vdots & \vdots \\
\alpha_{j-i-1}^{\prime} & \alpha_{j-i-1}^{\prime \prime} \\
\alpha_{j-i}^{\prime} & \alpha_{j-i}^{\prime \prime} \\
\gamma_{1}^{\prime} & \gamma_{1}^{\prime \prime} \\
\vdots & \vdots \\
\gamma_{i}^{\prime} & \gamma_{i}^{\prime \prime}
\end{array}\right), \ldots, \quad P_{j-1}=\left(\begin{array}{cc}
W^{\prime} & W^{\prime \prime} \\
\alpha_{1}^{\prime} & \beta_{1}^{\prime \prime} \\
\alpha_{2}^{\prime} & \beta_{2}^{\prime \prime} \\
\alpha_{3}^{\prime} & \beta_{3}^{\prime \prime} \\
\vdots & \vdots \\
\alpha_{j-i-1}^{\prime} & \beta_{j-i-1}^{\prime \prime} \\
\alpha_{j-i}^{\prime} & \alpha_{j-i}^{\prime \prime} \\
\gamma_{1}^{\prime} & \gamma_{1}^{\prime \prime} \\
\vdots & \vdots \\
\gamma_{i}^{\prime} & \gamma_{i}^{\prime \prime}
\end{array}\right) .
$$

Then $\left(P, P_{1}, \ldots, P_{j-1}, Q\right)$ is a path of length $j$. Since $\Gamma_{w}^{(m)}$ is a subgraph of $J_{q}(n+$ $l, m)$, the distance between $P$ and $Q$ is $j$.

Suppose $1<m \leq n-1$ and $l>0$. In order to determine the full automorphism group of $\Gamma_{w}^{(m)}$, we need to study its maximal cliques. It is direct to check that there are the following three types of maximal cliques:

(I) The set of $m$-dimensional subspaces in a given subspace of type $(m+1,0)$. Its size is $\left[\begin{array}{c}m+1 \\ 1\end{array}\right]$.

(II) The set of subspaces of type $(m, 0)$ in a given subspace of type $(m+1,1)$. By [13, Lemma 2.2] its size is $q^{m}$.

(III) The set $C(W)$ of subspaces of type $(m, 0)$ containing a given subspace $W$ of type $(m-1,0)$. By [13, Lemma 2.3] its size is $q^{l}\left[\begin{array}{c}n-m+1 \\ 1\end{array}\right]$.

Theorem 3.2 Let $1<m \leq n-1$ and $l>0$. Then the full automorphism group of the graph $\left(X_{m}, R_{0,1} \cup R_{1,0}\right)$ is $P \Gamma L\left(n+l, \mathbb{F}_{q}\right)_{E}$. 
Proof Let $f$ be an automorphism of the graph $\Gamma_{w}^{(m)}$. Then $f$ induces a permutation on the set of maximal cliques. Since the sizes of these three types of maximal cliques are pairwise distinct, $f$ induces a permutation on the set of maximal cliques of type III, and further induces a permutation $g$ on the set $X_{m-1}$ such that $f(C(W))=C(g(W))$, where $W$ is a subspace of type $(m-1,0)$.

Next, we shall show that $g$ is an automorphism of $\Gamma^{(m-1)}$. For any two vertices $W_{1}$ and $W_{2}$ of $\Gamma^{(m-1)}$, we can check that $W_{1}$ and $W_{2}$ are adjacent if and only if $C\left(W_{1}\right) \cap C\left(W_{2}\right) \neq \emptyset$. If $W_{1}$ and $W_{2}$ are adjacent, then

$$
f\left(C\left(W_{1}\right) \cap C\left(W_{2}\right)\right)=f\left(C\left(W_{1}\right)\right) \cap f\left(C\left(W_{2}\right)\right)=C\left(g\left(W_{1}\right)\right) \cap C\left(g\left(W_{2}\right)\right) \neq \emptyset,
$$

which implies that $g\left(W_{1}\right)$ and $g\left(W_{2}\right)$ are adjacent in $\Gamma^{(m-1)}$. So $g \in \operatorname{Aut}\left(\Gamma^{(m-1)}\right)$.

Similar to the proof of Theorem 2.6, $\operatorname{Aut}\left(\Gamma_{w}^{(m)}\right)$ is isomorphic to a subgroup of $\operatorname{Aut}\left(\Gamma^{(m-1)}\right)$. By Theorem 2.6, the desired result follows.

Acknowledgments The authors are indebted to the anonymous reviewers for their valuable suggestions and comments, which greatly improve the expression of this article. W. Liu's research is supported by NSFC $(11301138,11271004)$. K. Wang's research is supported by NSFC(11271047, 11371204) and the Fundamental Research Funds for the Central University of China.

\section{References}

1. Bannai, E., Ito, T.: Algebraic Combinatorics I: Association Schemes. The Benjamings/Cummings, Menlo Park (1984)

2. Brauer, R.: A characterization of null systems in projective space. Bull. Amer. Math. Soc. 42, 247-254 (1936)

3. Brouwer, A.E., Cohen, A.M., Neumaier, A.: Distance-Regular Graphs. Springer, Berlin (1989)

4. Chow, W.: On the geometry of algebraic homogeneous spaces. Ann. Math. 50, 32-67 (1949)

5. Deng, S., Li, Q.: On the affine geometry of algebraic homogeneous spaces. Acta Math. Sinica. 15, 651-663 (1965)

6. Fujisaki, T., Koolen, J.H., Tagami, M.: Some properties of the twisted Grassmann graphs. Innov. Incid. Geom. 3, 81-87 (2006)

7. Hua, L.: A theorem on matrices over a field and its applications. Acta Math. Sinica. 1, 109-163 (1951)

8. Kurihara, H.: Character tables of association schemes based on attenuated spaces. Ann. Comb. 17, 525-541 (2013)

9. van Dam, E.R., Koolen, J.H.: A new family of distance-regular graphs with unbounded diameter. Invent. Math. 162, 189-193 (2005)

10. Wan, Z.: Geometry of Matrices. World Scientific, Singapore (1996)

11. Wan, Z.: Geometry of Classical Groups over Finite Fields. Science Press, Bejing (2002)

12. Wang, K., Guo, J., Li, F.: Association schemes based on attenuated space. Eur. J. Comb. 31, 297-305 (2010)

13. Wang, K., Guo, J., Li, F.: Singular linear space and its application. Finite Fields Appl. 17, 395-406 (2011) 